\title{
Hodgkin's disease in an adult with acute lymphoblastic leukaemia
}

\author{
A. Zuiable, C. Borthwick-Clarke, J. Maitland, H. Clink and R. Powles \\ Department of Haematology and Leukaemia Unit, Royal Marsden Hospital, Downs Road, Sutton, Surrey \\ SM2 5PT, UK.
}

\begin{abstract}
Summary: A 53 year old woman with common acute lymphoblastic leukaemia (ALL) developed Hodgkin's disease 20 months after the initial diagnosis of ALL. This is by far the oldest case of Hodgkin's disease complicating ALL. The unusual presentation and the aetiology of Hodgkin's disease in ALL are discussed.
\end{abstract}

\section{Introduction}

Patients treated for acute lymphoblastic leukaemia (ALL) are now surviving long enough for the late effects of the disease and/or its therapy to emerge. Among these late complications second malignancies have been described (Ruymann \& Mosijezuk, 1981) including histiocytic medullary reticulosis, acute myeloid leukaemia, chronic myeloid leukaemia, solid tumours, polycythaemia and Hodgkin's disease.

The occurrence of acute leukaemia in patients being treated for Hodgkin's disease is well known (Coleman \& Williams, 1977). However, Hodgkin's disease developing in patients with ALL has only recently been described (Labotka, 1983) and is quite rare. There have been 7 cases reported to date, 4 of which were in children and 3 in young adults. We report here the first case of Hodgkin's disease in a middle-aged woman who was receiving maintenance therapy for ALL.

\section{Case report}

A 53 year old woman presented with a 6 week history of malaise and weight loss in April 1983. The peripheral white count was $14 \times 10^{9} / 1$ with $60 \%$ lymphoblasts. There was no lymphadenopathy or splenomegaly. Bone marrow aspirate confirmed the diagnosis of ALL L2 morphology (FAB criteria). Immunological markers indicated it was common ALL (82\% cALLA positive) and $80 \%$ TdT positive. Chromosome studies showed a normal female karyotype. She was treated with vincristine, prednisone, asparginase and daunorubicin according to the UKALL IX protocol and went into remission.

Correspondence: A. Zuiable, M.B., Ch.B.

Accepted: 31 October 1985
Cranial irradiation and intrathecal methotrexate were given for central nervous system prophylaxis and maintenance therapy of 6-mercaptopurine and methotrexate was started. She remained vaguely unwell with various complaints including nausea, occasional vomiting, weight loss and headaches. Extensive investigations revealed no abnormality. She continued to complain of vague symptoms. Physical examination was always normal.

In December 1984, 20 months after the initial diagnosis of ALL, she developed a painless swelling in the right groin and was found to have 2 enlarged lymph nodes. A biopsy showed mixed cellularity Hodgkin's disease with typical Reed-Sternberg cells, and lymphography showed extensive intra-abdominal nodal disease. Bone marrow aspirate and trephine were normal at this time. There was no significant lymphadenopathy above the diaphragm. Treatment with chlorambucil, vinblastine, prednisone and procarbazine has been started with marked clinical regresson of the disease.

\section{Discussion}

There are some interesting features in this case. This patient is by far the oldest reported case of Hodgkin's disease complicating ALL. The ages of the 7 patients previously reported ranged from 4 to 30 years and all but one were under 20 . The type of presentation of Hodgkin's disease in this patient is unusual in that the groin node presentation tends to be more common in males than in females.

The aetiology of Hodgkin's disease in ALL is unknown. The possibility of a constitutional tendency for multiple malignancies in some patients exists, or 
the origin may be extrinsic in that the carcinogen which caused the ALL may also cause the Hodgkin's disease or it may be due to chemotherapy. The incidence is too high to be due to chance alone.

The mean latent period between the diagnosis of ALL and Hodgkin's disease is 17 months (range 10-20 months) and, as many patients now survive beyond this, it is important to be aware of Hodgkin's disease as a possibility, especially as the presenting features may not be typical.

\section{References}

RUYMANN, F.B. \& MOSIJEZUK, A.D. (1981). Second malignancy in acute lymphocytic leukaemia. American Journal of Diseases of Children, 135, 313.

COLEMAN, C.N. \& WILLIAMS, C.J. (1977). Haematological

\section{Acknowledgement}

We thank Professor T.J. McElwain, Department of Medicine, Royal Marsden Hospital, Sutton, Surrey, for his help.

neoplasia in patients treated for Hodgkin's disease. New England Journal of Medicine, 297, 1249.

LABOTKA, R.J. (1983). Hodgkin's disease in a child with acute lymphoblastic leukaemia. Cancer, 52, 846. 